\begin{tabular}{|c|c|c|}
\cline { 2 - 3 } & REVISTA SABERES APUDEP & Volumen 3 Número 1 \\
\hline
\end{tabular}

\title{
PROCESO PARTICIPATIVO DE LA GESTIÓN DEL PROYECTO DE VINCULACIÓN: PROMOCIÓN INTEGRAL DE SALUD Y BIENESTAR CON INTERVENCIÓN DE TERAPIA FÍSICA EN PERSONAS CON DISCAPACIDAD FÍSICA
}

\author{
PARTICIPATORY PROCESS OF THE MANAGEMENT OF THE LINKAGE \\ PROJECT: INTEGRAL PROMOTION OF HEALTH AND WELFARE WITH THE \\ INTERVENTION OF PHYSICAL THERAPY IN PEOPLE WITH PHYSICAL \\ DISABILITIES
}

Ana Cristina Díaz Cevallos, Carolina Alexandra Paredes Jara y

Luis Felipe Arellano

Pontificia Universidad Católica del Ecuador. Email: anidiaz1208@hotmail.com, cpjalex@gmail.com; luifaf@yahoo.com

\section{RESUMEN}

Existe interés en los proyectos de vinculación e intervención para que las universidades del país promuevan la atención a sectores poblacionales carentes de recursos y apoyo. El Proyecto "Promoción Integral de Salud y Bienestar de la carrera de Terapia Física de la Pontificia Universidad Católica del Ecuador - Manos de Amor", es un ejemplo de ello. Se divulga el impacto de la implementación de una intervención fisioterapéutica individualizada y personalizada a 24 jóvenes del Barrio Jaime Roldós, en edades entre 18 y 28 años, con alteraciones neurológicas; los jóvenes forman parte de la Asociación Manos de Amor. Se brindó educación postural para la prevención de deformidades músculo-esqueléticas en pacientes con alteraciones neurológicas. Se disminuyeron los gastos de trasporte para asistencia a sesiones de fisioterapia.

PALABRAS CLAVE: Gestión de proyectos comunitarios, Terapia Física, Ecuador. 


\begin{tabular}{|c|c|c|}
\cline { 2 - 3 } & REVISTA SABERES APUDEP & Volumen 3 Número 1 \\
\hline
\end{tabular}

\begin{abstract}
There is interest in linking and intervention projects so that the country's universities promote attention to population sectors that lack resources and support. The Project "Integral Promotion of Health and Welfare of the Physical Therapy career of the Pontifical Catholic University of Ecuador - Manos de Amor", is one example of this. The impact of the implementation of an individualized and personalized physiotherapeutic intervention is disclosed to 24 young people of the Jaime Roldós neighborhood, aged between 18 and 28, with neurological alterations; the young people are part of the Hands of Love Association. Postural education was provided for the prevention of musculoskeletal deformities in patients with neurological disorders. Transportation expenses for attending physiotherapy sessions were reduced.
\end{abstract}

KEYWORDS: Management of community projects, Physical Therapy, Ecuador.

\title{
INTRODUCCIÓN
}

La Pontificia Universidad Católica del Ecuador (PUCE) continuamente apoya a la gestión de estudiantes y docentes que desarrollan actividades que tienen el propósito de lograr mejoras en la calidad de vida de un colectivo social vulnerable. Es a partir de la vinculación que la PUCE se convierte en una institución que transmite y aplica el conocimiento a la sociedad.

El proyecto Manos de Amor empezó en enero del 2018 con la idea de vincular a un sector ubicado a las afueras de Quito, específicamente en la comunidad de la RoldósPisulí en donde existe una gran cantidad de familias de escasos recursos que tienen familiares con discapacidad física con diferentes diagnósticos entre ellos parálisis cerebral, síndrome de Dawn, lesión medular, etc.

La participación en este proyecto de Vinculación con la Colectividad resulta ser un gran aprendizaje tanto personal como profesional para estudiantes y docentes, por el que se alcanzó: 


\begin{tabular}{|c|c|c|}
\cline { 2 - 3 } & REVISTA SABERES APUDEP & Volumen 3 Número 1 \\
\hline
\end{tabular}

Favorecer el desarrollo del proceso de enseñanza y aprendizaje entre estudiantes, docentes e investigadores que pongan en práctica todos los conceptos y metodologías aprendidas en el aula participando en la solución de problemas reales de sectores vulnerables.

Desarrollar programas de intervención en beneficio de la sociedad, a través de un plan estructurado y contribuir con la transmisión de un conocimiento validado.

Generar un ambiente de colaboración y comunicación para desarrollar formas de gestión para el progreso óptimo de la vinculación social.

Mejorar la manera de hacer servicio social, con un compromiso de los estudiantes, docentes e investigadores, tomando en cuenta que un pilar de PUCE es promover el servicio hacia los más necesitados.

\section{Marco Teórico}

La vinculación es el proceso integral que articula las funciones sustantivas de docencia, investigación y extensión de la cultura, así como los servicios de las instituciones de educación superior, para su interacción eficaz y eficiente con el entorno socioeconómico, mediante el desarrollo de acciones y proyectos de beneficio mutuo, que contribuyen a su posicionamiento y reconocimiento social (Trigueros, Berenice, \& Dávalos, 2016).

La Organización de las Naciones Unidas para la Educación, la Ciencia y la Cultura (UNESCO) recomienda a las universidades fortalecer las funciones de servicio a la sociedad, especialmente aquellas orientadas a erradicar la pobreza, la intolerancia, la violencia, el analfabetismo, el hambre, el deterioro del medio ambiente y las enfermedades (Editors-SAJCN, 2005). 


\begin{tabular}{|c|c|c|}
\cline { 2 - 3 } & REVISTA SABERES APUDEP & Volumen 3 Número 1 \\
\hline
\end{tabular}

Como establecen Rodríguez y Aristimuño (2014, 377): "la responsabilidad social universitaria está vinculada a las respuestas socialmente responsables o a los impactos que generan las IES en la sociedad y a su capacidad de difundir y practicar principios y valores éticos, por medio de procesos o funciones claves como lo son la gestión, la docencia, la investigación y la extensión".

Así mismo, la responsabilidad social de la educación superior es un pilar que la UNESCO señala como prioridad asumiendo el liderazgo social en la creación y aplicación de conocimientos fundamentados en la equidad, calidad y acceso.

En la creación y desarrollo del proyecto Manos de Amor se midió la percepción de todos los participantes: pacientes, estudiantes y docentes, por ello fue importante que dichos actores tuvieran claras sus funciones y sobre todo la responsabilidad social que conlleva la vinculación social. Responsabilidad social entendida como la forma de comprender la sociedad y actuar de forma comprometida.

\section{MATERIALES Y MÉTODOS}

El estudio realizado es de tipo observacional descriptivo cualitativo. El grupo de estudio lo conformaron 24 pacientes con trastornos neurológicos de: Síndrome de Down, traumatismo cráneo encefálico y parálisis cerebral, entre las edades de 18 a 28 años pertenecientes a la comunidad Jaime Roldós que forman parte de la Asociación Manos de Amor.

La participación de los estudiantes y docentes fue en el periodo del 27 de enero del 2018 al 25 de enero del 2019.

El total de estudiantes involucrados fue de 11, de sexto y séptimo nivel, pertenecientes a la carrera de licenciatura de Terapia Física de la Pontificia Universidad Católica del Ecuador, quienes en sus 160 horas de Vinculación Social, realizaron actividades de evaluación, diseño e implementación de un tratamiento fisioterapéutico. 


\begin{tabular}{|c|c|c|}
\cline { 2 - 3 } & REVISTA SABERES APUDEP & Volumen 3 Número 1 \\
\hline
\end{tabular}

Se midió el impacto social de la aplicación del Proyecto Social, mediante instrumentos cualitativos dirigidos a los 24 pacientes o actores sociales, a los 11 estudiantes participantes y los 3 docentes involucrados.

El primer instrumento cualitativo dirigido a los actores Sociales, consta de las siguientes preguntas:

1. El proyecto desarrollado generó cambios o mejoras en su comunidad (solucionó los problemas, ayudó con capacitación a las personas, etc.)

2. En el proyecto desarrollado se trabajó con los más necesitados de su comunidad, los integró al trabajo y les enseñó nuevas habilidades.

3. En el proyecto desarrollado participaron otras organizaciones como municipio, fundaciones y otros grupos sociales.

El segundo instrumento cualitativo dirigido a los estudiantes, consta de las siguientes preguntas:

1. La ejecución del programa y/o proyecto de Social comunitario (SC) que se realizó respondió a una planificación previamente definida.

2. En el programa y/o proyecto de SC realizado se priorizó el trabajo con los sectores más desfavorecidos promoviendo su desarrollo y superación, evitando prácticas asistencialistas o la instrumentalización de los mismos.

3. En el programa y/o proyecto de SC realizado se incorporó la participación de otros actores sociales y de saberes no académicos.

4. El programa y/o proyecto de SC realizado promovió la integración de diversas disciplinas como manera de abordar problemáticas complejas.

5. El programa y/o proyecto de SC realizado generó cambios o mejoras en la realidad de los beneficiarios (mediante la incorporación de nuevas soluciones, aumento de sus capacidades, etc.) 


\begin{tabular}{|c|c|c|}
\cline { 2 - 3 } & REVISTA SABERES APUDEP & Volumen 3 Número 1 \\
\hline
\end{tabular}

El tercer instrumento cualitativo dirigido a los docentes, consta de las siguientes preguntas:

1. La ejecución del programa y/o proyecto realizado respondió a una planificación previamente definida.

2. En el programa y/o proyecto realizado se priorizó el trabajo con los sectores más desfavorecidos promoviendo su desarrollo y superación, evitando prácticas asistencialistas o la instrumentalización de los mismos.

3. En el programa y/o proyecto realizado se incorporó la participación de otros actores sociales y de saberes no académicos.

4. El programa y/o proyecto realizado promovió la integración de diversas disciplinas como manera de abordar problemáticas complejas.

5. El programa y/o proyecto realizado generó cambios o mejoras en la docencia (mediante redefinición de mallas curriculares, nuevas carreras, etc.)

6. El programa y/o proyecto realizado generó cambios o mejoras en la investigación (mediante la articulación de nuevas líneas, proyectos, publicaciones, etc.)

7. El programa y/o proyecto realizado generó cambios o mejoras en la realidad de los beneficiarios (mediante la incorporación de nuevas soluciones, aumento de sus capacidades, etc.)

En todas estas encuestas, de actores sociales, estudiantes y docentes, tenían las opciones de seleccionar: Totalmente de acuerdo, De acuerdo, Neutro/Indeciso, En desacuerdo, Totalmente en desacuerdo, y No sabe/No contesta.

Todas las encuestas fueron tabuladas con el instrumento informático Statistical Product and Service Solutions (SPSS Versión 22 en español), herramienta de tratamiento de datos, se aplicó la opción de análisis de tablas de frecuencias y porcentajes. 


\begin{tabular}{|c|c|c|}
\cline { 2 - 3 } & REVISTA SABERES APUDEP & Volumen 3 Número 1 \\
\hline
\end{tabular}

\section{RESULTADOS Y DISCUSIÓN}

La Tabla 1 muestra los resultados en cuanto a la percepción de los actores sociales, sobre la gestión del proyecto de Vinculación Manos de Amor, el 90\% de los actores sociales beneficiados están Totalmente de acuerdo y el 10\% De acuerdo, que el proyecto generó cambios de la comunidad de Jaime Roldós, que, por cuestiones económicas, no se pueden trasladar con las personas en sillas de ruedas a centros especializados para recibir las sesiones de fisioterapia.

Tabla 1. Porcentajes de percepción de los actores sociales involucrados en el Proyecto de Vinculación- Manos de Amor

\begin{tabular}{|l|l|l|l|}
\hline Preguntas & $\begin{array}{l}\text { 1.El proyecto } \\
\text { desarrollado } \\
\text { generó cambios en } \\
\text { la comunidad }\end{array}$ & $\begin{array}{l}\text { 2. En el proyecto } \\
\text { desarrollado se } \\
\text { trabajó con los más } \\
\text { necesitados de su } \\
\text { comunidad, los } \\
\text { integró al trabajo y } \\
\text { les enseñó nuevas } \\
\text { habilidades. }\end{array}$ & $\begin{array}{l}\text { 3. En el proyecto } \\
\text { desarrollado } \\
\text { participaron otras } \\
\text { organizaciones } \\
\text { como municipio, } \\
\text { fundaciones y otros } \\
\text { grupos sociales. }\end{array}$ \\
\hline $\begin{array}{l}\text { Totalmente } \\
\text { de acuerdo }\end{array}$ & $90 \%$ & $100 \%$ & $85 \%$ \\
\hline De acuerdo & $10 \%$ & $0 \%$ & $15 \%$ \\
\hline Total & $100 \%$ & $100 \%$ & $100 \%$ \\
\hline
\end{tabular}

.Fuente: Informe de Resultados Cualitativos y Encuestas.

El $85 \%$ está totalmente de acuerdo en que el proyecto desarrollado participaron otras organizaciones como municipio, fundaciones y otros grupos sociales, ya que el municipio presta las instalaciones de Casa Somos Roldós- Pisulí para la realización de las sesiones de Fisioterapia y la policía Metropolitana de Quito también brinda el servicio de canoterapia e hipoterapia, una vez al mes. 


\begin{tabular}{|c|c|c|}
\cline { 2 - 3 } & REVISTA SABERES APUDEP & Volumen 3 Número 1 \\
\hline
\end{tabular}

El 100\% está totalmente de acuerdo en que el proyecto desarrollado se trabajó con los más necesitados de su comunidad, los integró al trabajo y les enseñó nuevas habilidades.

En lo que respecta a la percepción de los alumnos sobre la gestión del proyecto de Vinculación "Promoción Integral de Salud y Bienestar de intervención de Terapia Física - Manos de Amor", Tabla 2; el 100\% está totalmente de Acuerdo que en el proyecto realizado se priorizó el trabajo con los sectores más desfavorecidos promoviendo su desarrollo y superación, evitando prácticas asistencialistas o la instrumentalización de los mismos.

El 98\%, opina que está totalmente de acuerdo en que la ejecución del proyecto respondió a una planificación previamente definida. Esto posiblemente esté relacionado con la planificación responsable por parte de los docentes, la Dirección General de Vinculación de la Pontificia Universidad Católica del Ecuador, autoridades de la facultad de Enfermería y gestores de la Asociación Manos de Amor de la comunidad de Jaime Roldós, ubicada al noroccidente de Quito.

Está planificación, colaboración y participación activa de los estudiantes y docentes de Terapia Física, generó cambios o mejoras en la realidad de los beneficiarios mediante la intervención fisioterapéutica, generando aumento de sus capacidades neuromotrices, opina el $95 \%$ de los encuestados, al estar totalmente de acuerdo. 
REVISTA SABERES APUDEP

ISSN L 2644-3805

Acceso Abierto. Disponible en:

https://revistas.up.ac.pa/index.php/saberes_apudep
Volumen 3 Número 1

Enero-Junio 2020

Recibido: 08/09/2019

Aceptado: 06/12/2019

Tabla 2. Porcentajes de percepción de los estudiantes involucrados en el Proyecto de Vinculación- Manos de Amor

\begin{tabular}{|c|c|c|c|}
\hline Preguntas & $\begin{array}{l}\text { Totalmente } \\
\text { de acuerdo }\end{array}$ & $\begin{array}{l}\text { De } \\
\text { acuerdo }\end{array}$ & Total \\
\hline $\begin{array}{l}\text { La ejecución del proyecto respondió a una } \\
\text { planificación previamente definida. }\end{array}$ & $98 \%$ & $2 \%$ & $100 \%$ \\
\hline $\begin{array}{l}\text { En el proyecto realizado se priorizó el } \\
\text { trabajo con los sectores más } \\
\text { desfavorecidos promoviendo su } \\
\text { desarrollo y superación, evitando } \\
\text { prácticas asistencialistas o la } \\
\text { instrumentalización de los mismos. }\end{array}$ & $100 \%$ & $0 \%$ & $100 \%$ \\
\hline $\begin{array}{l}\text { En el programa y/o proyecto de } \mathrm{SC} \\
\text { realizado se incorporó la participación de } \\
\text { otros actores sociales y de saberes no } \\
\text { académicos. }\end{array}$ & $73 \%$ & $27 \%$ & $100 \%$ \\
\hline $\begin{array}{l}\text { El programa y/o proyecto de SC realizado } \\
\text { promovió la integración de } \\
\text { disciplinas como manera de } \\
\text { problemáticas complejas. }\end{array}$ & $83 \%$ & $17 \%$ & $100 \%$ \\
\hline $\begin{array}{l}\text { El programa y/o proyecto de SC realizado } \\
\text { generó cambios o mejoras en la realidad } \\
\text { de los beneficiarios (mediante la } \\
\text { incorporación de nuevas soluciones, } \\
\text { aumento de sus capacidades, etc.) }\end{array}$ & $95 \%$ & $5 \%$ & $100 \%$ \\
\hline
\end{tabular}

Fuente: Informe de Resultados de Encuestas.

Según los Testimonio de los estudiantes. "Al trabajar con los jóvenes que tienen síndrome de Down, secuelas de parálisis cerebral infantil, retraso mental (problemas neurológicos), con los cuales se les apoya tanto en el área de lenguaje como en el de terapia física, se les realiza técnicas enseñadas durante la carrera para ayudar a 


\begin{tabular}{|c|c|c|}
\cline { 2 - 3 } & REVISTA SABERES APUDEP & Volumen 3 Número 1 \\
\hline
\end{tabular}

movilizar las articulaciones y un mejor desenvolvimiento del paciente en su entorno social, dando resultados positivos, los pacientes colaboran para realizarles los ejercicios y todas las actividades que les realizamos durante la terapia que dura entre 30 y 40 minutos por paciente, el trabajo que se realiza es individual e integral lo que permitió evaluar las disfunciones psicomotrices, además se realizó primero una historia clínica con los datos que proporcionó la madre o la persona a cargo del paciente para tener una idea más clara de que se debe implantar en el plan de tratamiento específico para él o la paciente.

En cuanto a la percepción del docente, Tabla 3, se está totalmente de acuerdo (100\%) en los parámetros que el proyecto generó: El programa realizado generó mejoras en la docencia mediante apertura de sitio de práctica de otra asignatura de cuarto nivel de la carrera, incorporación del proyecto dentro del rediseño de la malla curricular en la asignatura de Fisioterapia Comunitaria, reestructuración de la cátedra de neurorrehabilitación en el rediseño y en la antigua malla curricular 2010, reforma de contenidos de las asignaturas de Psicomotricidad 1 y 2 de tercer y cuarto nivel. (Reforma curricular de la carrera de Fisioterapia, aprobada por el CES 2018) 
REVISTA SABERES APUDEP

ISSN L 2644-3805

Acceso Abierto. Disponible en:

https://revistas.up.ac.pa/index.php/saberes_apudep
Volumen 3 Número 1

Enero-Junio 2020

Recibido: 08/09/2019

Aceptado: 06/12/2019

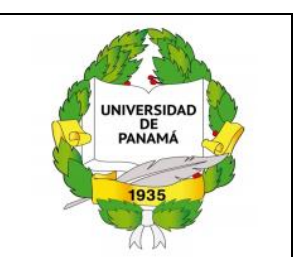

Tabla 3. Porcentajes de percepción de los docentes involucrados en el Proyecto de Vinculación- Manos de Amor

\begin{tabular}{|l|l|l|l|}
\hline Porcentajes Preguntas & $\begin{array}{l}\text { Totalmente } \\
\text { de acuerdo }\end{array}$ & $\begin{array}{l}\text { De } \\
\text { acuerdo }\end{array}$ & Total \\
\hline $\begin{array}{l}\text { La ejecución del programa y/o proyecto realizado } \\
\text { respondió a una planificación previamente } \\
\text { definida. }\end{array}$ & $100 \%$ & $0 \%$ & $100 \%$ \\
\hline $\begin{array}{l}\text { En el programa y/o proyecto realizado se priorizó } \\
\text { el trabajo con los sectores más desfavorecidos } \\
\text { promoviendo su desarrollo y superación, evitando } \\
\text { prácticas asistencialistas o la instrumentalización } \\
\text { de los mismos. }\end{array}$ & $100 \%$ & $0 \%$ & $100 \%$ \\
\hline $\begin{array}{l}\text { En el programa y/o proyecto realizado se } \\
\text { incorporó la participación de otros actores } \\
\text { sociales y de saberes no académicos. }\end{array}$ & $67 \%$ & $33 \%$ & $100 \%$ \\
\hline $\begin{array}{l}\text { El programa y/o proyecto realizado promovió la } \\
\text { integración de diversas disciplinas como manera } \\
\text { de abordar problemáticas complejas. }\end{array}$ & $100 \%$ & $0 \%$ & $100 \%$ \\
\hline $\begin{array}{l}\text { El programa y/o proyecto realizado generó } \\
\text { cambios o mejoras en la docencia (mediante } \\
\text { redefinición de mallas curriculares, nuevas } \\
\text { carreras, etc.) }\end{array}$ & $100 \%$ & $0 \%$ & $100 \%$ \\
\hline $\begin{array}{l}\text { El programa y/o proyecto realizado generó } \\
\text { cambios o mejoras en la investigación (mediante } \\
\text { la articulación de nuevas líneas, proyectos, } \\
\text { publicaciones, etc.) }\end{array}$ & $67 \%$ & $33 \%$ & $100 \%$ \\
\hline $\begin{array}{l}\text { El programa y/o proyecto realizado generó } \\
\text { cambios o mejoras en la realidad de los } \\
\text { beneficiarios (mediante la incorporación de } \\
\text { nuevas soluciones, aumento de sus capacidades, } \\
\text { etc.) }\end{array}$ & $100 \%$ & $0 \%$ \\
\hline
\end{tabular}

Fuente: Informe de estudiantes y de Resultados Cualitativos y Encuestas 


\begin{tabular}{|c|c|c|c|}
\cline { 2 - 3 } & REVISTA SABERES APUDEP & Volumen 3 Número 1 \\
\hline
\end{tabular}

Además se logró la incorporación de otras carreras de salud al proyecto comunitario. El proyecto también ha permitido la escritura de este artículo científico y la proyección de dos tesis doctorales en Salud.

\section{CONCLUSIÓN}

Las universidades tienen un papel relevante en la construcción y transmisión de conocimientos cuyo fin es promover nuevos escenarios que eleven la calidad de vida de los sectores más vulnerables. La docencia y la investigación son actividades que continuamente realiza la universidad a través de los programas de vinculación social que constituye el pilar fundamental para proporcionar una participación responsable y comprometida de todos sus participantes.

Los programas de vinculación son una estrategia bidireccional, pues como docente permite formar estudiantes que tengan conocimientos sólidos, pero sobretodo valores bioéticos para la aplicación del proyecto de vinculación de manera sistemática, es decir con un proceso de evaluación, diagnóstico, tratamiento fisioterapéutico y medidas de cuidado postural, como forma de prevención de deformidades músculoesqueléticas y trastornos asociados en personas con alteraciones neurológicas; y como estudiante, contar con una formación integral.

Toda la implementación y desarrollo del proyecto Manos de Amor fue totalmente adecuada pues permitió cambios y mejoras en la comunidad, percibida, tanto por los actores sociales, docentes y estudiantes siendo el resultado de una planificación previamente definida por todos los involucrados en el programa.

El proyecto además generó cambios y mejoras en las reformas curriculares de la carrera de Licenciatura en Fisioterapia de la Pontificia Universidad Católica del Ecuador, con la proyección de tesis doctorales y producción científica de artículos. 


\begin{tabular}{|c|c|c|}
\cline { 2 - 3 } & REVISTA SABERES APUDEP & Volumen 3 Número 1 \\
\hline
\end{tabular}

\section{RECOMENDACIÓN}

La universidad debe mantener vinculación permanente con la sociedad y definir las vías por medio de las cuales debe poner su conocimiento y capacidad de acción al servicio de las comunidades más necesitadas como objetivo primordial para de esta manera enfocar un proyecto con sustentabilidad en el tempo.

No simplemente se requiere la generación de proyectos de intervención, también es indispensable la gestión social de estos proyectos, orientada a la consecución y ejecución de programas con la intensión de impactar en la mejora tanto de la institución como de la sociedad siempre enfocándose en procesos de formación, investigación, liderazgo social y compromiso que son los elementos fundamentales para hacer efectiva su incidencia social.

Es importante mencionar que la gestión social realizada incorpore un aprendizaje conjunto, continuo y actualizado para todos los participantes para que en un futuro permita incidir en el diseño de las políticas públicas.

\section{REFERENCIAS BIBLIOGRÁFICAS}

Beltran-Llevador Jose, Iñigo-Bajo Enrique y Mata-Segreda Alejandrina (2014). "La responsabilidad social universitaria, el reto de su construcción permanente", en Revista Iberoamericana de Educación Superior (RIES), México, UNAMIISUE/Universia, vol. V, Núm. 14, pp. 3-18. Recuperado de http://ries.universia.net/index.php/ries/article/view/367

Editors - SAJCN. (2005). The glycaemic index - scientific evidence on the practical use. S Afr J Clin Nutr, 18(3), 211-212. https://doi.org/ED.2009/CONF.402/2 Conferencia

Paper, C. (2017). La Responsabilidad Social Universitaria en el ámbito de la Universidad Siglo 21, (October 2016).

Rodríguez Monroy, Carlos, Aristimuño, Minerva, (2014) Responsabilidad social universitaria. Su gestión desde la perspectiva de directivos y docentes. Estudio 


\begin{tabular}{|c|c|c|}
\cline { 2 - 3 } & REVISTA SABERES APUDEP & Volumen 3 Número 1 \\
\hline
\end{tabular}

de caso: una pequeña universidad latinoamericanalnterciencia [en linea] 2014, 39 (Junio-Junio) : [Fecha de consulta: 9 de mayo de 2016] Disponible en:<http://www.redalyc.org/articulo.oa?id=33931213003> ISSN 0378-1844

Trigueros, A. B., Berenice, M., \& Dávalos, M. (2016). Vinculación social : Acciones, visiones y estrategias desde la universidad para y con la sociedad, 56-65.

UNESCO (2009). "La Responsabilidad Social de la Educación Superior", en Conferencia Mundial de la Educación Superior. Paris.

Vallaeys Fançois (2008). "¿Qué es la Responsabilidad Social Universitaria?”. Recuperado de: http://www.ausjal.org/tl_files/ausjal/images/contenido/Documentos/Publicacione s/Educacion\%20

superior/Que\%20es\%20la\%20Responsabilidad\%20Social\%20Universitaria\%20

- \%20Francois\%20Vallaeys.pdf 